\title{
Complete integrability of a modified vector derivative nonlinear Schrödinger equation
}

\author{
Ralph Willox ${ }^{1}$, Willy Hereman ${ }^{2}$ and Frank Verheest ${ }^{3}$ \\ ${ }^{1}$ Dienst Theoretische Natuurkunde, Vrije Universiteit Brussel, \\ Pleinlaan 2, B-1050 Brussel, Belgium \\ ${ }^{2}$ Department of Mathematical and Computer Sciences, \\ Colorado School of Mines, \\ Golden, Colorado 80401-1887, USA \\ ${ }^{3}$ Sterrenkundig Observatorium, Universiteit Gent, \\ Krijgslaan 281, B-9000 Gent, Belgium
}

October 29, 2018

\begin{abstract}
Oblique propagation of magnetohydrodynamic waves in warm plasmas is described by a modified vector derivative nonlinear Schrödinger equation, if charge separation in Poisson's equation and the displacement current in Ampère's law are properly taken into account. This modified equation cannot be reduced to the standard derivative nonlinear Schrödinger equation and hence its possible integrability and related properties need to be established afresh. Indeed, the new equation is shown to be integrable by the existence of a bi-Hamiltonian structure, which yields the recursion operator needed to generate an infinite sequence of conserved densities. Some of these have been found explicitly by symbolic computations based on the symmetry properties of the new equation. Since the new equation includes as a special case the derivative nonlinear Schrödinger equation, the recursion operator for the latter one is now readily available.
\end{abstract}




\section{Introduction}

The derivative nonlinear Schrödinger equation (DNLS) was first given by Rogister (1971) for the nonlinear evolution of parallel Alfvén waves in plasmas, and later encountered in many different contexts by other authors, emerging as one of the canonical nonlinear equations in physics. The DNLS could also account for slightly oblique propagation of Alfvén waves (see e.g. Hada, Kennel and Buti 1989), albeit at the price of neglecting two effects which might be important in strongly magnetized astrophysical plasmas. One is the deviation from charge neutrality between the different plasma species, the other is the influence of the displacement current in Ampère's law. Retaining these effects results in a nonlinear vector evolution equation which differs from the standard vector form of the DNLS by an extra linear term, and therefore was called the modified vector derivative nonlinear Schrödinger equation (MVDNLS) (Deconinck, Meuris and Verheest 1993a, 1993b).

Recently, there has been a renewed interest in the use of the DNLS for certain astrophysical plasmas (Spangler 1992, Spangler and Plapp 1992), assuming that the case of slightly oblique propagation could easily be reduced to that of parallel propagation. To do so, one modifies the dependent variable, in this case the perpendicular magnetic field, by including the static part as well. Since the MVDNLS cannot be transformed into the DNLS itself, such an easy transition from parallel to oblique propagation is not possible. The reverse is true, of course, the MVDNLS includes the DNLS as a special case, when we go from oblique to parallel propagation, which amounts to dropping the bothersome extra term.

That term, which distinguishes the MVDNLS from the DNLS, has implications for the discussion of integrability and the possibility of deriving solitary wave solutions for the MVDNLS. The DNLS is well known to be completely integrable (Kaup and Newell 1978), whereas for the MVDNLS we could only get certain indications about its integrability (Deconinck, Meuris and Verheest 1993b). The applicability of the prolongation method (Kaup 1980), adapted to a vector nonlinear equation, and the existence of some invariants (Deconinck, Meuris and Verheest 1993b) were indicative of complete integrability, without giving a watertight proof.

In the present paper we show that the MVDNLS possesses a bi-Hamiltonian structure, and hence through the resulting recursion operator an infinite sequence of conserved densities. Interestingly enough, to the best of our knowledge there seems to be no proof in the literature that the DNLS itself has a bi-Hamiltonian structure, although this expected property is mentioned sometimes without further details nor references (Oevel and 
Fuchssteiner 1992). The existence of an infinite sequence of conserved densities is proved by Kaup and Newell (1978) for the DNLS, without showing the bi-Hamiltonian character. Our constructions, formulas and conclusions for the MVDNLS immediately apply to the DNLS. Hence, without extra work, the explicit forms of both Hamiltonians and the recursion operator for the DNLS are now available.

In $\S 2$ we recall the form of the MVDNLS and list in $\S 3$ some of the conserved densities, which were found in an ad hoc fashion with the help of a symbolic program and by looking at the symmetry properties of the equation. The knowledge of these conserved densities turned out to be beneficial for an easy construction of the appropriate Hamiltonians in $\S 4$. In $\S 5$ the bi-Hamiltonian structure and the recursion operator are derived and with it we established the existence of an infinite sequence of conserved densities, needed to guarantee complete integrability. $\S 6$ is devoted to a short discussion of the implications for the usual DNLS, and in $\S 7$ we draw some conclusions.

\section{MVDNLS}

The MVDNLS is, after the necessary scaling and Galilean transforms to cast it in its simplest dimensionless form, given by

$$
\frac{\partial \mathbf{B}_{\perp}}{\partial t}+\frac{\partial}{\partial x}\left(B_{\perp}^{2} \mathbf{B}_{\perp}\right)+\alpha \mathbf{B}_{\perp 0} \mathbf{B}_{\perp 0} \cdot \frac{\partial \mathbf{B}_{\perp}}{\partial x}+\mathbf{e}_{x} \times \frac{\partial^{2} \mathbf{B}_{\perp}}{\partial x^{2}}=\mathbf{0},
$$

where the parameter $\alpha$ characterizes the extra term which distinguishes the MVDNLS from the DNLS. In (1) $\mathbf{B}_{\perp}$ stands for the perpendicular magnetic field, which includes both the wave contributions and the static perpendicular field due to the oblique propagation with respect to the total external magnetic field. The direction of wave propagation is along the $x$-axis. If the third term is absent, we can project (1) onto axes perpendicular to the direction of wave propagation, introduce a new complex variable from the components of $\mathbf{B}_{\perp}$,

$$
\phi_{ \pm}=B_{y} \pm i B_{z}
$$

and combine the projections to obtain the DNLS in standard scalar form,

$$
\frac{\partial \phi_{ \pm}}{\partial t}+\frac{\partial}{\partial x}\left(\left|\phi_{ \pm}\right|^{2} \phi_{ \pm}\right) \pm i \frac{\partial^{2} \phi_{ \pm}}{\partial x^{2}}=0
$$

The \pm signs in (2) and (3) are correlated. The DNLS can account for oblique propagation, provided $\alpha$ is zero, i.e. imposing charge neutrality to all orders and neglecting the displacement current in Ampère's law (Deconinck, Meuris and Verheest 1993a). The 
bothersome third term in (1) also disappears if $\mathbf{B}_{\perp 0}$ is zero, in the case of strictly parallel propagation.

At this stage, it is worth recalling that the DNLS has constant-amplitude solutions of the form

$$
\begin{aligned}
& B_{y}=a \cos (k x-\omega t), \\
& B_{z}= \pm a \sin (k x-\omega t),
\end{aligned}
$$

with $a$ an arbitrary constant. As can easily be checked, there are no constant amplitude solutions to (1) besides the trivial case with $\mathbf{B}_{\perp}=\mathbf{B}_{\perp 0}$, if $\mathbf{B}_{\perp 0} \neq 0$. This means that any sort of separation into left and right circularly polarized waves as for the DNLS is doomed to fail. Of course, for oblique propagation with $\alpha=0$, the circular polarization given by the DNLS is only apparent, since $\mathbf{B}_{\perp}$ includes the static part $\mathbf{B}_{\perp 0}$, and this shift leads in reality to elliptical polarization for the perpendicular wave field (Spangler and Plapp 1992). In addition, the MVDNLS has a class of stationary solitary wave solutions which the DNLS does not have, the subalfvénic modes, which have totally different properties compared to the known stationary solutions of the DNLS (Deconinck, Meuris and Verheest 1993b).

We know that the DNLS is completely integrable in the sense that it is possesses an infinite series of conserved densities, which can be constructed explicitly. As the MVDNLS reduces for $\alpha \rightarrow 0$ to the vector form of the DNLS, there is hope to prove integrability for the MVDNLS. There are, however, two major complications with the MVDNLS: it has a vector character which contrary to the DNLS cannot be transformed away, and the boundary values at infinity are not zero (at least not in the physical model for which the nonlinear evolution equation was derived!).

One encounters in the literature quite a variety of methods to investigate symmetries, to construct conservation laws, or to establish integrability of nonlinear equations via direct or inverse methods. While in principle these methods could be used, one rarely sees worked examples involving vector equations. Furthermore, the nonzero boundary conditions for the MVDNLS are an additional hurdle.

\section{$3 \quad$ Integrability and conserved densities}

The first step in any treatment is to try to prove integrability, or at least collect sufficiently compelling evidence. For instance, one could check if the equation passes the Painlevé test. 
However, here again the nonscalar character of the MVDNLS prevents straightforward application of this otherwise so useful test (Fordy 1990). A different way to ascertain integrability, at least as convincing, is indicated by Kaup (1980) and Fordy (1990). It involves the application of the prolongation method due to Estabrook and Wahlquist. This method was adapted successfully to the nonlinear equation at hand (Deconinck, Meuris and Verheest 1993b).

Yet another important tool to determine integrability of a nonlinear PDE is finding a sufficiently large number of conservation laws of the form

$$
\frac{\partial \rho}{\partial t}+\frac{\partial J}{\partial x}=0
$$

where $\int_{-\infty}^{+\infty} \rho d x$ is the conserved quantity with density $\rho$ and associated flux $J$. We have assumed functions $u$ and $v$ which are fast decreasing at infinity to symmetric but in the case of $u$ non-zero boundary conditions. As is well known from many other examples, the first conservation law comes from rewriting the equations themselves in the form of a conservation law. For the MVDNLS this yields after projection

$$
\begin{aligned}
& u_{t}+\left(u\left(u^{2}+v^{2}\right)+\beta u-v_{x}\right)_{x}=0, \\
& v_{t}+\left(v\left(u^{2}+v^{2}\right)+u_{x}\right)_{x}=0,
\end{aligned}
$$

where $u$ and $v$ denote the components of $\mathbf{B}_{\perp}$ parallel and perpendicular to $\mathbf{B}_{\perp 0}$, and $\beta=\alpha B_{\perp 0}^{2}$. As usual, subscripts refer to partial derivatives with respect to $t$ and $x$. Whereas (6) amounts to a vector conservation law, the other ones we have derived through constructive procedures are all scalar ones, involving powers of $\left(u^{2}+v^{2}\right)$, as presented in (9)-(13).

To see how to proceed, we follow ideas exposed in more detail in Verheest and Hereman (1994), and briefly discuss the scaling or symmetry properties of the equations. These can be used to obtain information about polynomial conserved densities and the building blocks they are made off. The scaling of (6) is such that

$$
u \sim v, \quad \frac{\partial}{\partial t} \sim \frac{\partial^{2}}{\partial x^{2}}, \quad u^{2} \sim v^{2} \sim \beta \sim \frac{\partial}{\partial x}
$$

We may restrict ourselves to building blocks which belong to the same class under the mentioned scaling, since for any mixed conserved quantity which one could derive, the freedom implied in the scaling would split that quantity in several conserved quantities, each with building blocks of the same scaling. Thus there is a straightforward and logical way to construct invariant quantities (Miura, Gardner and Kruskal (1968)). Moreover and without loss of generality, we may remove any density (or part thereof) that is a 
total $x$-derivative, for these are trivially conserved. In addition, for $\beta=0$ (6) is invariant under the substitution

$$
u \rightarrow v, \quad v \rightarrow-u
$$

In every conserved density the part without factors $\beta$ will have to obey this additional symmetry.

At the quadratic level in $u$ and $v$, the only possibility is $u^{2}+v^{2}$ or (9) given below, due to the rule in (8). Cubic terms in $u$ and $v$ are not possible at all.

Starting then with a candidate density containing the building block $\left(u^{2}+v^{2}\right)^{2}$, one has four factors $u$ or $v$, and one could add a combination of the form $u^{3} v-u v^{3}$. Keeping the scaling (7) in mind, quartic terms are also equivalent to two factors $u$ and/or $v$ and one derivation, or to two factors $u$ and/or $v$ and one factor $\beta$. Two factors $u$ and/or $v$ with one derivation can only lead to a non-trivial building block of the structure $u v_{x}-v u_{x}$, if we keep all the preceding remarks in mind. With one factor $\beta$ we could in principle have a linear combination of $u^{2}, u v$ and $v^{2}$. This exhausts the building blocks at this order and it is then for the MATHEMATICA program we wrote to determine the necessary coefficients. This leads to (10). Obviously, we can go on like this for higher orders.

Labelling the conserved densities $\rho_{n}$, with $n$ the corresponding power of $\left(u^{2}+v^{2}\right)$, we obtained

$$
\begin{aligned}
\rho_{1}= & u^{2}+v^{2}, \\
\rho_{2}= & \frac{1}{2}\left(u^{2}+v^{2}\right)^{2}-u v_{x}+u_{x} v+\beta u^{2}, \\
\rho_{3}= & \frac{1}{4}\left(u^{2}+v^{2}\right)^{3}+\frac{1}{2}\left(u_{x}^{2}+v_{x}^{2}\right)-u^{3} v_{x}+v^{3} u_{x}+\frac{\beta}{4}\left(u^{4}-v^{4}\right), \\
\rho_{4}= & \frac{1}{4}\left(u^{2}+v^{2}\right)^{4}-\frac{2}{5}\left(u_{x} v_{x x}-u_{x x} v_{x}\right)+\frac{4}{5}\left(u u_{x}+v v_{x}\right)^{2} \\
& +\frac{6}{5}\left(u^{2}+v^{2}\right)\left(u_{x}^{2}+v_{x}^{2}\right)-\left(u^{2}+v^{2}\right)^{2}\left(u v_{x}-u_{x} v\right) \\
& +\frac{\beta}{5}\left(2 u_{x}^{2}-4 u^{3} v_{x}+2 u^{6}+3 u^{4} v^{2}-v^{6}\right)+\frac{\beta^{2}}{5} u^{4}, \\
& \frac{7}{16}\left(u^{2}+v^{2}\right)^{5}+\frac{1}{2}\left(u_{x x}^{2}+v_{x x}^{2}\right)-\frac{5}{2}\left(u^{2}+v^{2}\right)\left(u_{x} v_{x x}-u_{x x} v_{x}\right)+5\left(u^{2}+v^{2}\right)\left(u u_{x}+v v_{x}\right)^{2} \\
& +\frac{15}{4}\left(u^{2}+v^{2}\right)^{2}\left(u_{x}^{2}+v_{x}^{2}\right)^{2}-\frac{35}{16}\left(u^{2}+v^{2}\right)^{3}\left(u v_{x}-u_{x} v\right) \\
& +\frac{\beta}{8}\left(5 u^{8}+10 u^{6} v^{2}-10 u^{2} v^{6}-5 v^{8}+20 u^{2} u_{x}^{2}-12 u^{5} v_{x}+60 u v^{4} v_{x}-20 v^{2} v_{x}^{2}\right) \\
& +\frac{\beta^{2}}{4}\left(u^{6}+v^{6}\right) .
\end{aligned}
$$


Note that we have not yet included the renormalization constants needed to ensure the boundedness of the conserved quantities obtained from the above listed densities. We will come back to this point in the following paragraph.

\section{Hamiltonian structure}

Let us start by pointing out some of the principal ingredients of the Hamiltonian structure of evolution equations, adapted where necessary to vector quantities.

The system (6) is said to possess a Hamiltonian structure (Olver 1980), if there exists a so called Hamiltonian operator $\Theta$ (Fokas 1987) (sometimes called implectic operator, see e.g. Fuchssteiner and Fokas 1981) and a (2-component) gradient vector function $\gamma_{H}(u, v)$ such that (6) can be written in the form:

$$
\left(\begin{array}{l}
u \\
v
\end{array}\right)_{t}=\Theta \cdot \gamma_{H}
$$

The operator $\Theta$ is a Hamiltonian operator if it is skew-symmetric

$$
\langle\Theta \cdot \mathbf{a}, \mathbf{b}\rangle=-\langle\mathbf{a}, \Theta \cdot \mathbf{b}\rangle
$$

with respect to the scalar product

$$
\langle\mathbf{f}, \mathbf{g}\rangle=\int_{-\infty}^{+\infty} \mathbf{f}(x) \cdot \mathbf{g}(x) d x
$$

and if it satisfies a "Jacobi-like" identity. The precise form (A.1) of that identity is given in the Appendix, since it is not of immediate importance here. A vector function $\gamma_{H}(u, v)$ is a gradient function if its Fréchet derivative is symmetric with respect to the scalar product (16):

$$
\left\langle\gamma_{H}^{\prime}[\mathbf{a}], \mathbf{b}\right\rangle=\left\langle\mathbf{a}, \gamma_{H}^{\prime}[\mathbf{b}]\right\rangle
$$

Recall that the Fréchet derivative of a vector function $\gamma_{H}(u, v)=\left(\begin{array}{c}\gamma_{1}(u, v) \\ \gamma_{2}(u, v)\end{array}\right)$ in a $\operatorname{direction}\left(\begin{array}{c}\xi \\ \eta\end{array}\right)$ is given by

$$
\gamma_{H}^{\prime}\left[\left(\begin{array}{c}
\xi \\
\eta
\end{array}\right)\right]=\left.\frac{\partial}{\partial \varepsilon}\left(\begin{array}{c}
\gamma_{1}(u+\varepsilon \xi, v)+\gamma_{1}(u, v+\varepsilon \eta) \\
\gamma_{2}(u+\varepsilon \xi, v)+\gamma_{2}(u, v+\varepsilon \eta)
\end{array}\right)\right|_{\varepsilon=0} .
$$

The Hamiltonian, or Hamiltonian functional, $H=\int_{-\infty}^{+\infty} \rho(u, v) d x$ giving rise to the gradient function $\gamma_{H}(u, v)$ through the identity (Berger 1977)

$$
H^{\prime}[\boldsymbol{\zeta}]=\int_{-\infty}^{+\infty} \rho^{\prime}[\boldsymbol{\zeta}] d x \equiv\left\langle\boldsymbol{\gamma}_{H}, \boldsymbol{\zeta}\right\rangle
$$


can be recovered from this gradient in the following way:

$$
H=\int_{0}^{1}\left[\left\langle\gamma_{H}(\lambda u, \lambda v),\left(\begin{array}{l}
u \\
v
\end{array}\right)\right\rangle-C\right] d \lambda
$$

where the constant $C$ is chosen such that the integral giving $H$ is bounded. Such a Hamiltonian formulation admits a Poisson bracket,

$$
\{A, B\} \equiv\left\langle\gamma_{A}, \Theta \cdot \gamma_{B}\right\rangle
$$

defining the time evolution of a functional $A$ of $u$ and $v$ :

$$
\begin{aligned}
\frac{d A}{d t}=\frac{\partial A}{\partial t}+A^{\prime}\left[\left(\begin{array}{l}
u \\
v
\end{array}\right)_{t}\right] & =\frac{\partial A}{\partial t}+\left\langle\gamma_{A},\left(\begin{array}{c}
u \\
v
\end{array}\right)_{t}\right\rangle \\
& =\frac{\partial A}{\partial t}+\left\langle\gamma_{A}, \Theta \cdot \gamma_{H}\right\rangle=\frac{\partial A}{\partial t}+\{A, H\}
\end{aligned}
$$

using formulas (19) and (14).

Since $\Theta$ is skew-symmetric, and due to identity (A.1), the bracket (21) possesses all the characteristics of a standard Poisson bracket, except for the "Leibniz-like" expulsion property which cannot be properly defined in the case of functionals. Clearly, if autonomous, the Hamiltonian $H$ itself is a conserved quantity for the evolution equation (14). Since (6) can be written as a conservation law, an apparent Hamiltonian formulation is the following:

$$
\begin{gathered}
\left(\begin{array}{l}
u \\
v
\end{array}\right)_{t}=\Theta_{2} \cdot \gamma_{2}, \\
\Theta_{2}=-\left(\begin{array}{cc}
\partial_{x} & 0 \\
0 & \partial_{x}
\end{array}\right) \quad, \quad \gamma_{2}=\left(\begin{array}{c}
\left(u^{2}+v^{2}\right) u-v_{x}+\beta u \\
\left(u^{2}+v^{2}\right) v+u_{x}
\end{array}\right),
\end{gathered}
$$

where $\partial_{x}$ denotes the partial derivative with respect to $x$.

$\Theta_{2}$ is easily seen to be a Hamiltonian operator since it is a constant (i.e. not depending on $u$ or $v$ ), skew-symmetric operator (see Appendix).

It is straightforward to verify that $\gamma_{2}$ satisfies (17) and thus leads to a Hamiltonian functional $\mathrm{H}_{2}$ given by formula (20):

$$
\begin{aligned}
H_{2} & =\int_{0}^{1} d \lambda \int_{-\infty}^{+\infty}\left[\lambda^{3}\left(u^{2}+v^{2}\right)^{2}+\lambda\left(u_{x} v-u v_{x}+\beta u^{2}\right)-C_{2}\right] d x \\
& =\int_{-\infty}^{+\infty}\left[\frac{\left(u^{2}+v^{2}\right)^{2}}{4}+\frac{u_{x} v-u v_{x}}{2}+\frac{\beta}{2} u^{2}-C_{2}\right] d x
\end{aligned}
$$


Comparison with formula (10) shows that it is the conserved density $\rho_{2}$ which give rise to this Hamiltonian. One may wonder if the conserved density $\rho_{1}$ in (9) can be linked to a Hamiltonian functional as well. Let us define $H_{1}$ by

$$
H_{1}=\int_{-\infty}^{+\infty}\left[\frac{\rho_{1}}{2}-C_{1}\right] d x=\int_{-\infty}^{+\infty}\left[\frac{u^{2}+v^{2}}{2}-C_{1}\right] d x
$$

Using (19), the gradient $\boldsymbol{\gamma}_{1}$ of this functional is found to be:

$$
\gamma_{1}=\left(\begin{array}{l}
u \\
v
\end{array}\right)
$$

The challenge is to find a Hamiltonian operator $\Theta_{1}$ such that (6) can be recast into the form (14) using $\gamma_{1}$. The MVDNLS equation would then have a second Hamiltonian formulation and thus possess a bi-Hamiltonian structure.

\section{$5 \mathrm{Bi}-$ Hamiltonian structure and recursion operator}

If an evolution equation admits a bi-Hamiltonian formulation (Magri 1978)

$$
\left(\begin{array}{l}
u \\
v
\end{array}\right)_{t}=\Theta_{1} \cdot \gamma_{1}=\Theta_{2} \cdot \gamma_{2}
$$

and if it is possible to show that $\Theta_{1}$ and $\Theta_{2}$ are compatible Hamiltonian operators (i.e. $\Theta_{1}+\Theta_{2}$ is again a Hamiltonian operator), and if one of the operators, say $\Theta_{2}$ is invertible, then the operator

$$
R=\Theta_{1} \cdot \Theta_{2}^{-1}
$$

is a hereditary recursion operator (Fuchssteiner 1979, Fuchssteiner and Fokas 1981) for that evolution equation.

The formal adjoint of $R$ with respect to the scalar product (16)

$$
R^{\dagger}=\Theta_{2}^{-1} \cdot \Theta_{1}
$$

then maps gradients of conserved quantities into gradients, provided the operator $R$ is injective (Fuchssteiner and Fokas 1981, and also Appendix), thus defining an infinite sequence of conserved quantities, all in involution with respect to the Poisson bracket $(21)$.

In our case we have already found one Hamiltonian structure with an invertible Hamiltonian operator $\Theta_{2}$ :

$$
\Theta_{2}^{-1}=-\left(\begin{array}{cc}
\partial_{x}^{-1} & 0 \\
0 & \partial_{x}^{-1}
\end{array}\right)
$$


where $\partial_{x}^{-1}$ denotes the inverse of the $\partial_{x}$ operator, such that $\partial_{x} \partial_{x}^{-1}=\partial_{x}^{-1} \partial_{x}=1$.

A first step towards finding a second Hamiltonian structure is the construction of a skew-symmetric operator $\Theta_{1}$ which casts (6) into Hamiltonian form with the gradient $\gamma_{1}$ in (26). The most general parametrization (involving a single $\partial_{x}^{-1}$ operator) which satisfies the above constraints is

$$
\Theta_{1}=-\left(\begin{array}{ll}
\theta_{11} & \theta_{12} \\
\theta_{21} & \theta_{22}
\end{array}\right)
$$

with

$$
\begin{aligned}
& \theta_{11}=\beta \partial_{x}+c_{1} v \partial_{x} v+c_{3} u \partial_{x} u+\left(6-4 c_{3}\right) u_{x} \partial_{x}^{-1} u_{x}, \\
& \theta_{12}=-\partial_{x}^{2}+\left(\frac{3}{2}-\frac{c_{1}+c_{2}}{2}-\frac{c_{5}}{4}\right) u v \partial_{x}+\left(\frac{1}{2}-\frac{c_{1}-c_{2}}{2}+\frac{c_{5}}{4}\right) u v_{x} \\
& +\left(1-c_{1}-\frac{c_{5}}{2}\right) u_{x} v+c_{5} u_{x} \partial_{x}^{-1} v_{x} \\
& \theta_{21}=\partial_{x}^{2}+\left(\frac{3}{2}-\frac{c_{1}+c_{2}}{2}-\frac{c_{5}}{4}\right) u v \partial_{x}+\left(\frac{1}{2}+\frac{c_{1}-c_{2}}{2}+\frac{c_{5}}{4}\right) u_{x} v \\
& +\left(1-c_{1}-\frac{c_{5}}{2}\right) u v_{x}+c_{5} v_{x} \partial_{x}^{-1} u_{x}, \\
& \theta_{22}=c_{2} u \partial_{x} u+c_{4} v \partial_{x} v+\left(6-4 c_{4}\right) v_{x} \partial_{x}^{-1} v_{x} \text {. }
\end{aligned}
$$

At this point one could of course try to find values of the parameters for which this operator is actually a Hamiltonian operator. However, this proves to be a formidable task (see Appendix). A better approach is to concentrate on the action of $R^{\dagger}$ on the gradient functions obtained so far. Because of the bi-Hamiltonian structure (27) of the equations, $R^{\dagger}$ maps $\gamma_{1}$ into $\gamma_{2}$ :

$$
\gamma_{2}=\Theta_{2}^{-1} \cdot \Theta_{1} \cdot \gamma_{1}=R^{\dagger} \cdot \gamma_{1}
$$

Suppose $R^{\dagger}$ actually is the formal adjoint of a recursion operator for the MVDNLS equation, then it will map $\gamma_{2}$ into yet another gradient function. If the conserved quantity corresponding to this gradient has to be polynomial in $u, v$ and their derivatives, then the values of the parameters in (32) have to be such that $\Theta_{1} \cdot \gamma_{2}$ is a total $x$-derivative:

$$
\gamma_{3}=\Theta_{2}^{-1} \cdot \Theta_{1} \cdot \gamma_{2}=-\left(\begin{array}{cc}
\partial_{x}^{-1} & 0 \\
0 & \partial_{x}^{-1}
\end{array}\right) \cdot \Theta_{1} \cdot \gamma_{2}
$$

This requirement uniquely determines all five parameters in (32), namely $c_{1}=c_{2}=0, c_{3}=$ $c_{4}=-c_{5}=2$, thus giving us a single candidate for a second Hamiltonian operator:

$$
\Theta_{1} \equiv-\left(\begin{array}{cc}
\beta \partial_{x}+2 u \partial_{x} u-2 u_{x} \partial_{x}^{-1} u_{x} & -\partial_{x}^{2}+2 v \partial_{x} u-2 u_{x} \partial_{x}^{-1} v_{x} \\
\partial_{x}^{2}+2 u \partial_{x} v-2 v_{x} \partial_{x}^{-1} u_{x} & 2 v \partial_{x} v-2 v_{x} \partial_{x}^{-1} v_{x}
\end{array}\right)
$$

together with the gradient

$$
\gamma_{3}=\beta \boldsymbol{\gamma}_{2}+\left(\begin{array}{c}
\beta u^{3}-u_{x x}+\frac{3}{2} u^{5}+\frac{3}{2} u v^{4}+3 u^{3} v^{2}-3 v^{2} v_{x}-3 u^{2} v_{x} \\
-\beta v^{3}-v_{x x}+\frac{3}{2} v^{5}+\frac{3}{2} u^{4} v+3 u^{2} v^{3}+3 u_{x} v^{2}+3 u^{2} u_{x}
\end{array}\right)
$$


Redefining $\gamma_{3}$ as $\gamma_{3}-\beta \gamma_{2}$, it is straightforward to show that it satisfies (17) and corresponds to the Hamiltonian functional

$$
H_{3}=\int_{-\infty}^{+\infty}\left[\frac{\left(u^{2}+v^{2}\right)^{3}}{4}+u_{x} v^{3}-u^{3} v_{x}-\frac{1}{2}\left(u u_{x x}+v v_{x x}\right)+\frac{\beta}{4}\left(u^{4}-v^{4}\right)-C_{3}\right] d x .
$$

The density function associated with this functional is, up to partial integration, the conserved density $\rho_{3}$ given in (11). Hence, it follows that $R^{\dagger}$ maps $\gamma_{2}$ into the gradient of a conserved quantity (i.e., $H_{3}+\beta H_{2}$ ), suggesting that $R$ indeed is a hereditary recursion operator for the MVDNLS equation.

In the Appendix it is proven that $\Theta_{1}$ is a Hamiltonian operator and that it is compatible with $\Theta_{2}$. Thus, we have shown that

$$
R=\left(\begin{array}{cc}
\beta+2 u^{2}+2 u_{x} \partial_{x}^{-1} u & -\partial_{x}+2 u v+2 u_{x} \partial_{x}^{-1} v \\
\partial_{x}+2 u v+2 v_{x} \partial_{x}^{-1} u & 2 v^{2}+2 v_{x} \partial_{x}^{-1} v
\end{array}\right)
$$

which follows from (28), (30) and (35), is a hereditary recursion operator for the MVDNLS equation:

$$
\left(\begin{array}{l}
u \\
v
\end{array}\right)_{t}=\Theta_{1} \cdot \Theta_{2}^{-1} \cdot\left(\begin{array}{l}
u \\
v
\end{array}\right)_{x}=R \cdot\left(\begin{array}{l}
u \\
v
\end{array}\right)_{x} .
$$

The recursion operator therefore defines a hierarchy of integrable evolution equations

$$
\left(\begin{array}{l}
u \\
v
\end{array}\right)_{t}=R^{n} \cdot\left(\begin{array}{l}
u \\
v
\end{array}\right)_{x}
$$

all sharing an infinite sequence of conserved quantities, the gradients of which are generated by the formal adjoint of $R$ :

$$
R^{\dagger}=\left(\begin{array}{cc}
\beta+2 u \partial_{x}^{-1} u \partial_{x} & -\partial+2 u \partial_{x}^{-1} v \partial_{x} \\
\partial_{x}+2 v \partial_{x}^{-1} u \partial_{x} & 2 v \partial_{x}^{-1} v \partial_{x}
\end{array}\right)
$$

\section{The DNLS as special case}

For $\beta=0$ we recover from the MVDNLS the usual DNLS itself. Hence $\left.R\right|_{\beta=0}$ or

$$
R=\left(\begin{array}{cc}
2 u^{2}+2 u_{x} \partial_{x}^{-1} u & -\partial_{x}+2 u v+2 u_{x} \partial_{x}^{-1} v \\
\partial_{x}+2 u v+2 v_{x} \partial_{x}^{-1} u & 2 v^{2}+2 v_{x} \partial_{x}^{-1} v
\end{array}\right)
$$

is the recursion operator for DNLS, since it can be seen that the $\beta$ term in $\Theta_{1}$ does not alter the fact that it is a Hamiltonian operator (see Appendix). The Hamiltonian formulation (23) reduces to the one given by Kaup and Newell (1978) when $\beta=0$. However, to the best of our knowledge, the second Hamiltonian formulation is a new result, not found in the literature, and neither is the recursion operator (42). 


\section{Conclusions}

Slightly oblique propagation of Alfvén waves in strongly magnetized plasmas is described by a nonlinear vector evolution equation which differs from the vector form of the DNLS by an extra linear term, if one retains both the deviation from charge neutrality between the different plasma species and the influence of the displacement current in Ampère's law. The resulting modified vector derivative nonlinear Schrödinger equation cannot be transformed into the DNLS itself, and this has implications for the discussion of integrability and the possibility of finding conserved densities and solitary wave solutions. Of course, the reverse is true: the MVDNLS includes the DNLS as a special case.

While in a previous paper the applicability of the prolongation method, adapted to a vector nonlinear equation, and the existence of some invariants were indicative of complete integrability, in the present paper we have shown that the MVDNLS indeed possesses a bi-Hamiltonian structure, and hence, through the resulting recursion operator, an infinite sequence of conserved densities. We were guided in this by the explicit symbolic computation of the first seven conserved densities.

Surprisingly enough, there seems to be no proof in the literature that the DNLS itself has a bi-Hamiltonian structure, although the existence of an infinite sequence of conserved densities was known. As the DNLS emerges as a special case of the MVDNLS studied here, we now also have the explicit form of the recursion operator for the DNLS, besides the proof of its bi-Hamiltonian structure.

\section{Acknowledgements}

The National Fund for Scientific Research (Belgium) is thanked for a special research grant, which made the stay of WH at the Universiteit Gent possible, during which this

work was initiated. One of the authors (RW) is a Senior Research Assistant at the National Fund for Scientific Research (Belgium). WH acknowledges support of the National Science Foundation of America under Grant CCR-9300978. 


\section{Appendix}

A skew-symmetric operator $\Theta$ is a Hamiltonian operator if and only if it satisfies the identity (Fuchssteiner and Fokas 1981)

$$
\left\langle\mathbf{a}, \Theta^{\prime}[\Theta \cdot \mathbf{c}] \cdot \mathbf{b}\right\rangle+\left\langle\mathbf{b}, \Theta^{\prime}[\Theta \cdot \mathbf{a}] \cdot \mathbf{c}\right\rangle+\left\langle\mathbf{c}, \Theta^{\prime}[\Theta \cdot \mathbf{b}] \cdot \mathbf{a}\right\rangle \equiv 0
$$

where the scalar product was defined in (16). Since this identity involves taking the Fréchet derivative of $\Theta$ in a certain direction, it is trivially satisfied if $\Theta$ does not depend upon $u$ or $v$, i.e. if it is a "constant" operator. Hence, every constant skew-symmetric operator is a Hamiltonian operator.

Verifying that $\Theta_{1}$ satisfies (A.1) is a very cumbersome task. A method which makes such a verification a lot more tractable relies on defining a functional multivector (see Olver 1986)

$$
\mathcal{Z}=\frac{1}{2} \int\left\{(\xi \eta) \wedge \Theta \cdot\left(\begin{array}{l}
\xi \\
\eta
\end{array}\right)\right\} d x .
$$

Using this formalism, it can be shown (Olver 1986) that the identity (A.1) can be recast into the form

$$
\mathcal{Z}^{\prime}\left[\Theta \cdot\left(\begin{array}{c}
\xi \\
\eta
\end{array}\right)\right] \equiv 0
$$

assuming, by definition, that $\xi$ and $\eta$ are independent of $u, v$ or their derivatives.

In our case we define the multivector

$$
\begin{aligned}
\mathcal{Z}_{1}=\frac{1}{2} \int & \left\{\left(\beta+2 u^{2}\right) \xi \wedge \xi_{x}-2 u_{x} \xi \wedge \partial_{x}^{-1} u_{x} \xi-2 \xi \wedge \eta_{x x}+4 u v \xi \wedge \eta_{x}\right. \\
& \left.+4 u_{x} v \xi \wedge \eta-4 u_{x} \xi \wedge \partial_{x}^{-1} v_{x} \eta+2 v^{2} \eta \wedge \eta_{x}-2 v_{x} \eta \wedge \partial_{x}^{-1} v_{x} \eta\right\} d x
\end{aligned}
$$

It is a straightforward, albeit tedious, calculation to show that

$$
\mathcal{Z}_{1}^{\prime}\left[\Theta_{1} \cdot\left(\begin{array}{l}
\xi \\
\eta
\end{array}\right)\right]=0
$$

thus proving that $\Theta_{1}$ is a Hamiltonian operator.

In the same manner it can be shown that

$$
\mathcal{Z}_{1}^{\prime}\left[\Theta_{2} \cdot\left(\begin{array}{l}
\xi \\
\eta
\end{array}\right)\right]=0
$$

and hence that $\Theta_{1}+\Theta_{2}$ is a Hamiltonian operator as well, i.e. thus $\Theta_{1}$ and $\Theta_{2}$ are compatible Hamiltonian operators. Note that since the only term in $\mathcal{Z}_{1}$ depending on $\beta$ 
is independent of $u$ or $v$, its presence (or absence) does not alter the properties (A.5) or (A.6). Consequently, the above considerations also hold in the special case of the standard DNLS equation.

Concerning the injective nature of the recursion operator $R$, which is needed to guarantee that $R^{\dagger}$ maps gradients to gradients (see Proposition 2 in Fuchssteiner and Fokas 1981), it suffices to consider the action of $R$ on the space $\mathcal{S}_{P}$ of (2-component) vector functions with polynomial components in $u, v$ and their derivatives, which are all $\mathcal{C}^{\infty}$ functions fast decreasing at infinity to symmetric but in the case of $u$ non-zero boundary conditions. It is easily seen that the kernel of $R$ in this space consists of the elements which are of zero polynomial degree. Since $R$ either raises the polynomial degree of the different terms by two or leaves it invariant, $R$ can be made injective by restricting the domain to the space $\mathcal{S}_{P_{0}} \subset \mathcal{S}_{P}$ without zero degree elements. 


\section{References}

Berger M S 1977, Nonlinearity and Functional Analysis (Academic Press, N Y)

Deconinck B, Meuris P and Verheest F 1993a, J. Plasma Phys. 50, 445-455

Deconinck B, Meuris P and Verheest F 1993b, J. Plasma Phys. 50, 457-476

Fokas A S 1987, Stud. in Appl. Math. 77, 253-299

Fordy A 1990, in: Soliton theory: a survey of results (edited by A Fordy, Manchester University Press, Manchester) 403-426

Fuchssteiner B 1979, Nonlin. Anal., Theor. Meth. \& Appl. 3, 849-862

Fuchssteiner B and Fokas A S 1981, Physica D 4, 47-66

Hada T, Kennel C F and Buti B 1989, J. Geophys. Res. 94, 65-77

Kaup D J 1980, Physica D 1, 391-411

Kaup D J and Newell A C 1978, J. Math. Phys. 19, 798-801

Magri F 1978, J. Math. Phys. 19, 1156-1162

Miura R M, Gardner C S and Kruskal M D 1968, J. Math. Phys. 9, 1204-1209

Oevel G and Fuchssteiner B 1992, Physica A 181, 364-384

Olver P J 1980, Proc. Camb. Phil. Soc. 88, 71-88

Olver P J 1986, Applications of Lie Groups to Differential Equations (Springer, N Y)

Rogister A 1971, Phys. Fluids 14, 2733-2739

Spangler S R 1992, in: Solar Wind Seven (edited by E Marsch and R Schwenn, Pergamon Press, Oxford) 539-544

Spangler S R and Plapp B B 1992, Phys. Fluids B 4, 3356-3370

Verheest F and Hereman W 1994, Physica Scripta, in press 